\title{
ON THE NATURAL GENERALIZATION OF THE NATURAL CONDITIONS OF LADYZHENSKAYA AND URAL'TSEVA
}

\author{
GARY M. LIEBERMAN
}

Department of Mathematics, Iowa State University, Ames, Iowa 50011, USA

0. Introduction. Consider the problem of minimizing the functional

$$
\mathcal{I}[u]=\int_{\Omega} F(x, u, D u) d x
$$

over some class of real-valued functions on the domain $\Omega \subset \mathbb{R}^{n}$. To answer basic questions about the minima, such as existence, uniqueness, and regularity, appropriate structure must be placed on the function $F$ and on the underlying function space in which the minimization occurs.

In the simple case $F(x, z, p)=\left(1+|p|^{2}\right)^{m / 2}$, where $m>1$ is a constant, Ladyzhenskaya and Ural'tseva [21] proved complete results. A key element of their analysis is that the Euler-Lagrange equation for $\mathcal{I}$ is uniformly elliptic. We write this equation as

$$
\operatorname{div}\left(\left(1+|D u|^{2}\right)^{(m-2) / 2} D u\right)=0
$$

or as

$$
a^{i j}(D u) D_{i j} u=0
$$

where

$$
a^{i j}(p)=\left(1+|p|^{2}\right)^{(m-2) / 2}\left[\delta^{i j}+\frac{(m-1) p^{i} p^{j}}{1+|p|^{2}}\right] .
$$

Uniform ellipticity means that the eigenvalues of the matrix $\left(a^{i j}\right)$ are positive and the ratio of maximum to minimum eigenvalue is bounded independently of $p$. Another element of their analysis is the correct function space for minimization, the standard Sobolev space $W^{1, m}$, which is a separable reflexive Banach space in which $C^{\infty}$ is dense.

To understand the role of uniform ellipticity, we seek the most general structure on $F$ which leads to a uniformly elliptic Euler-Lagrange equation. If 
$F(x, z, p)=G(|p|)$, this structure is easy to identify. Writing $g=G^{\prime}$, the EulerLagrange equation is

$$
\operatorname{div}\left(g(|D u|) \frac{D u}{|D u|}\right)=0
$$

or $(0.1)$ with

$$
a^{i j}(p)=\frac{g(|p|)}{|p|}\left[\delta^{i j}+\left\{\frac{g^{\prime}(|p|)|p|}{g(|p|)}-1\right\} \frac{p^{i} p^{j}}{|p|^{2}}\right] .
$$

Therefore the Euler-Lagrange equation is elliptic if and only if $g^{\prime}>0$ and uniformly elliptic if and only if there are positive constants $\delta \leq g_{0}$ such that

$$
\delta \leq g^{\prime}(t) t / g(t) \leq g_{0} .
$$

(This structure condition was first brought to my attention as the example in [34, p. 844].) In this case, we use $W^{1, G}$, the space of weakly differentiable functions $u$ with $G(|D u|) \in L^{1}$, in place of $W^{1, m}$. $W^{1, G}$ is also a separable, reflexive Banach space in which $C^{\infty}$ is dense (because (0.2) implies that the defining function for the appropriate Orlicz space satisfies a $\Delta_{2}$ condition, see [18] for details). Various technical difficulties arise in using $W^{1, G}$, however. They are based primarily on the nonhomogeneity of $G$.

To see that the class of functions satisfying (0.2) includes functions which are not asymptotically power functions, we let $\alpha, \beta, \varepsilon$ be constants with $\beta>\alpha>\varepsilon$ $>0$ and we define the sequence $\left(t_{k}\right)$ by $t_{0}=2, t_{k}=\left(t_{k-1}\right)^{(\beta+\varepsilon-\alpha) / \varepsilon}$. The function $g$ given by

$$
g(t)= \begin{cases}t^{\alpha}, & 1 \leq t<t_{0}, \\ \left(t_{2 k}\right)^{\alpha-\beta-\varepsilon} t^{\beta+\varepsilon}, & t_{2 k} \leq t<t_{2 k+1}, \\ \left(t_{2 k+1}\right)^{\beta-\alpha+\varepsilon} t^{\alpha-\varepsilon}, & t_{2 k+1} \leq t<t_{2 k+2}, \\ 1 / g(1 / t), & t<1,\end{cases}
$$

satisfies (0.2) with $\delta=\alpha-\varepsilon$ and $g_{0}=\beta+\varepsilon$. Moreover,

$$
\begin{aligned}
& \limsup _{t \rightarrow \infty} t^{-\theta} g(t)= \begin{cases}\infty & \text { if } \theta<\beta, \\
1 & \text { if } \theta=\beta, \\
0 & \text { if } \theta>\beta,\end{cases} \\
& \liminf _{t \rightarrow \infty} t^{-\theta} g(t)= \begin{cases}\infty & \text { if } \theta<\alpha, \\
1 & \text { if } \theta=\alpha, \\
0 & \text { if } \theta>\alpha,\end{cases}
\end{aligned}
$$

with similar behavior as $t \rightarrow 0^{+}$. In fact, this $g$ is only piecewise $C^{1}$, but this point can be handled in any number of ways, as we shall see.

In addition, (0.2) is satisfied for $g(t)=t^{m-1}$ with $\delta=g_{0}=m-1$, for $g(t)=t\left(1+t^{2}\right)^{(m-2) / 2}$ with $\delta=\min \{1, m-1\}$ and $g_{0}=\max \{1, m-1\}$, and for $g(t)=t^{m-1} \ln (t+1)$ with $\delta=m-1$ and $g_{0}=m$ provided $m>1$. (More specifically, $t g^{\prime}(t) / g(t)$ goes monotonically from 1 to $m-1$ as $t$ goes from 0 to $\infty$ in the second case and from $m$ to $m-1$ in the third.) 
1. Known results for quasilinear elliptic equations. As we previously mentioned, much of the analysis of the functional $\mathcal{I}$ is based on a study of quasilinear elliptic equations. Accordingly, we now switch to this setting and study the operator $Q$ defined by

$$
Q u=\operatorname{div} A(x, u, D u)+B(x, u, D u)
$$

for functions $A$ and $B$ satisfying the following natural conditions of Ladyzhenskaya and Ural'tseva [21] (also known as the Leray-Lions conditions):

$$
\begin{gathered}
p \cdot A(x, z, p) \geq|p|^{m}-a_{1}(x)|z|^{m}-a_{2}(x), \\
|A(x, z, p)| \leq a_{3}|p|^{m-1}+a_{4}(x)|z|^{m-1}+a_{5}(x), \\
|B(x, z, p)| \leq b_{0}|p|^{m}+b_{1}(x)|p|^{m-1}+b_{2}(x)|z|^{m}+b_{3}(x)
\end{gathered}
$$

for nonnegative constants $a_{3}$ and $b_{0}$, and nonnegative functions $a_{1}, a_{2}, a_{4}, a_{5}, b_{1}$, $b_{2}, b_{3}$ in suitable $L^{p}$ spaces.

Ladyzhenskaya and Ural'tseva showed that any weak solution $u$ of $Q u=0$ in $\Omega$, which is in $W^{1, m}$, is bounded if these conditions hold with $b_{0}=0$. They also showed that bounded solutions are Hölder continuous even for nonzero $b_{0}$. Under the additional hypothesis that $A$ is locally Lipschitz with

$$
\begin{gathered}
|p|^{2}\left|A_{p}\right|+|p|\left|A_{z}\right|+\left|A_{x}\right|=O\left(|p|^{m}\right) \quad \text { as }|p| \rightarrow \infty, \\
\left(a^{i j}\right) \geq \lambda\left(1+|p|^{2}\right)^{(m-2) / 2} I,
\end{gathered}
$$

where here $I$ is the identity matrix and $\lambda$ is a positive constant, bounded solutions are $C^{1, \alpha}$ for some positive $\alpha$. They also proved corresponding results up to the boundary provided one of the boundary conditions

$$
\begin{gathered}
u=\phi \quad \text { on } \partial \Omega, \\
A(x, u, D u) \cdot \gamma=\psi(x, u) \quad \text { on } \partial \Omega
\end{gathered}
$$

(with $\gamma$ the unit inner normal) is satisfied for sufficiently smooth $\partial \Omega$, $\phi$, and $\psi$. Optimal regularity results were proved by Giaquinta and Giusti [14] for (1.4a), namely $\phi \in C^{1, \beta}, \partial \Omega \in C^{1, \beta}$ implies $u \in C^{1, \alpha}$ (assuming, of course, all the previously given structure conditions on $Q$ ). The present author [24] showed that $\psi \in C^{\beta}$ and $\partial \Omega \in C^{1, \beta}$ implies $u \in C^{1, \alpha}$ for (1.4b). In both cases $\alpha=\beta$ if $\beta$ is small enough or $A_{p}$ is continuous with respect to $p$.

When the hypotheses on $A_{p}$ are modified to

$$
\lambda|p|^{m-2} I \leq\left(a^{i j}\right) \leq \Lambda|p|^{m-2} I
$$

for positive constants $\lambda \leq \Lambda$, the equation becomes degenerate wherever $D u=0$. Nonetheless, Uhlenbeck [36] and Ural'tseva [37] proved interior $C^{1, \alpha}$ regularity in this case (and also for systems) under restricted hypotheses. Their methods and results were extended to the full structure by DiBenedetto [4], Tolksdorf [35], and others. Boundary $C^{1, \alpha}$ regularity is due to the present author [24].

When $|p|^{m}$ is replaced by $G(|p|)$, or, equivalently, by $|p| g(|p|)$, in these structure conditions, various elements of these known results no longer apply. In particular, 
the work of Ladyzhenskaya and Ural'tseva uses the Sobolev imbedding of $W^{1, m}$ in $L^{n m /(n-m)}$ for $m<n$. A corresponding imbedding theorem for $W^{1, G}$ was proved by Donaldson and Trudinger [12], but the application of this imbedding theorem to $L^{\infty}$ bounds for arbitrary $g$ satisfying (0.2) was done only very recently by Korolev [17], and a proof of Hölder estimates by these means is yet to be seen.

In the next section, we shall see that all these regularity results follow from the simplest Sobolev imbedding of $W^{1,1}$ in $L^{n /(n-1)}$.

2. Pointwise estimates for the general structure conditions. We now present the basic estimates for sub- and supersolutions of $Q u=0$ when $t^{m}$ is replaced by $t g(t)$ in the structure conditions. Complete proofs appear in [26]. The basic structure conditions are

$$
\begin{gathered}
p \cdot A \geq|p| g(|p|)-a_{1} g\left(\frac{|z|}{R}\right) \frac{|z|}{R}-a_{2}, \\
|A| \leq a_{3} g(|p|)+a_{4} g\left(\frac{|z|}{R}\right)+a_{5}, \\
z B \leq b_{0}|p| g(|p|)+b_{1} \frac{|z|}{R} g\left(\frac{|z|}{R}\right)+b_{2}
\end{gathered}
$$

for nonnegative constants $a_{1}, a_{2}, a_{3}, a_{4}, a_{5}, b_{0}, b_{1}, b_{2}$, and a positive constant $R$. (More generally, $a_{1}, a_{2}, a_{4}, a_{5}, b_{1}, b_{2}$ may lie in certain $L^{p}$ classes as described in [26].) We use $\chi$ to denote a constant such that

$$
a_{2}+b_{2} \leq \chi g(\chi), \quad a_{5} \leq g(\chi),
$$

and we write $u^{+}$for the positive part of $u$.

THEOREM 2.1. If $Q u \geq 0$ in $B(R)$, a ball of radius $R$, and if conditions (2.1) are satisfied, then for any $p>0$, there is a constant $C_{p}$ determined only by $a_{1}$, $a_{3}, a_{4}, b_{0}, b_{1}, g_{0}, n$, and $p$ such that

$$
\sup _{B(R / 2)} u \leq C_{p}\left[\left(R^{-n} \int_{B(R)}\left(u^{+}\right)^{p} d x\right)^{1 / p}+\chi R\right] .
$$

Pr o of. Set $\bar{u}=u^{+}+\chi R$; then $\bar{u}$ satisfies the differential inequality $\bar{Q} \bar{u} \geq 0$ for some $\bar{Q}$ satisfying our structure conditions with $a_{2}=a_{5}=b_{2}=0$ and $a_{1}, a_{4}$, and $b_{1}$ increased by 1 . With $q$ and $r$ suitably chosen constants, $\eta$ a standard cut-off function, and $v=\eta \bar{u} / R$, we use $G(v)^{q-1} \eta^{r} \bar{u}$ as test function in the weak form of $\bar{Q} \bar{u} \geq 0$. Setting $\theta=2+2 g_{0}$, we find that

$$
\int_{B(R)} \eta^{r} G(v)^{q-1}|D u| g(|D u|) d x \leq C[1+|r|+|q|] \int_{B(R)} \eta^{r-\theta} G(v)^{q} d x
$$

By direct calculation, we have

$$
|D G(v)| \leq \frac{\eta}{R}|D \bar{u}| g(v)+|D \eta| \frac{\bar{u}}{R} g(v)
$$


note that there is an underlying philosophy that $D \bar{u} \approx \bar{u} / R$ and $D \eta \approx \eta / R$. These very rough approximations are one of the new elements in the proof. The next is a sort of Young's inequality: because $g$ is increasing we have

$$
a g(b) \leq a g(a) \quad \text { if } a \geq b, \quad a g(b) \leq b g(b) \quad \text { if } a \leq b
$$

and hence

$$
a g(b) \leq a g(a)+b g(b) .
$$

Choosing $a=|D \bar{u}|$ and $b=v$, we see that

$$
\begin{aligned}
\int_{B(R)} \eta^{r-1} G(v)^{q-1}|D G(v)| d x \leq \frac{1}{R} & \int \eta_{B(R)}^{r} G(v)^{q-1}|D u| g(|D u|) d x \\
& +\frac{1+g_{0}}{R} \int_{B(R)} \eta^{r} G(v)^{q} d x+\frac{1+g_{0}}{R} \int_{B(R)} \eta^{r-2}(R|D \eta|) G(v)^{q} d x .
\end{aligned}
$$

By choosing $\eta$ so that $|D \eta| \leq 2 / R$ and setting $\kappa=n /(n-1)$, from these inequalities and Sobolev's inequality we obtain

$$
\left(\int_{B(R)} \eta^{\kappa(r-1)} G(v)^{\kappa q} d x\right)^{1 / \kappa} \leq C[1+|r|+|q|]^{2} \frac{1}{R} \int_{B(R)} \eta^{r-\theta} G(v)^{q} d x
$$

If we choose $r$ so that $\kappa(r-1)=r-\theta$, a standard iteration argument yields

$$
\sup _{B(R)} G(v) \leq C_{q}\left[R^{-n} \underset{B(R)}{\int} \eta^{-n(\theta-1)} G(v)^{q} d x\right]^{1 / q}
$$

for any $q>0$. Recalling that $G(v) \leq v g(v) \leq\left(1+g_{0}\right) G(v)$, we see for $q=n(\theta-1)$ that

$$
\begin{aligned}
& \sup _{B(R)} v \leq C\left[R^{-n} \int_{B(R)} \eta^{-q} G(v)^{q} d x\right]^{1 / q} / \sup _{B(R)} g(v) \\
& \leq C\left[R^{-n} \underset{B(R)}{\int} \eta^{-q} v^{q} d x\right]^{1 / q}=\frac{C}{R}\left[R^{-n} \int_{B(R)} \bar{u}^{q} d x\right]^{1 / q} .
\end{aligned}
$$

This inequality easily implies $(2.3)$ for $p=n(\theta-1)$ and an interpolation argument gives the result for arbitrary $p>0$.

An intermediate result called the weak Harnack inequality is used to prove Hölder continuity of bounded solutions. Since we assume $u$ to be bounded, we may consider the structure conditions

$$
\begin{gathered}
p \cdot A \geq|p| g(|p|)-a_{2}, \quad|A| \leq a_{3} g(|p|)+a_{5}, \\
-z B \leq b_{0}|p| g(|p|)+b_{2} .
\end{gathered}
$$

TheOrem 2.2. If $Q u \leq 0$ and $u \geq 0$ in $B(R)$, and if conditions (2.4) are satisfied, there are positive constants $C$ and $p$ determined only by $a_{3}, b_{0}, \delta, g_{0}$, 
and $n$ such that

$$
\left(R^{-n} \int_{B(2 R / 3)} u^{p} d x\right)^{1 / p} \leq C\left[\inf _{B(R / 2)} u+\chi R\right] .
$$

Proof. With $\chi$ and $\bar{u}$ as before, it is not hard to show that

$$
\left(R^{-n} \int_{B(2 R / 3)} \bar{u}^{-s} d x\right)^{1 / s} \leq C_{s} \inf _{B(R / 2)} \bar{u}
$$

for any $s>0$. To finish the proof we need to show that

$$
\int_{B(\varrho)}|D(\ln \bar{u})| d x \leq C p^{n-1}
$$

for any ball $B(\varrho)$ (not necessarily concentric with $B(R)$ ) such that $B(2 \varrho) \subset B(R)$. From this inequality, it follows by the John-Nirenberg inequality that

$$
\left(\int_{B(2 R / 3)} \bar{u}^{p} d x\right)\left(\int_{B(2 R / 3)} \bar{u}^{-p} d x\right) \leq C R^{2 n}
$$

for some $p>0$. Combining this inequality with (2.6) for $s=p$ gives (2.5).

The proof of (2.7) is fairly simple. Let $\eta$ be a cut-off function in $B(2 \varrho)$ and use $G(\bar{u} / \varrho)^{-1} \eta^{g_{0}} \bar{u}$ as test function. A little calculation gives

$$
\int_{B(2 \varrho)} \frac{|D \bar{u}| g(|D \bar{u}|)}{G(\bar{u} / \varrho)} \eta^{g_{0}} d x \leq C \varrho^{n} .
$$

Moreover,

$$
\begin{aligned}
\int_{B(\varrho)}|D(\ln \bar{u})| d x & =\int_{B(\varrho)} \frac{|D \bar{u}|}{\bar{u}} d x=\frac{1}{\varrho} \int_{B(\varrho)} \frac{|D \bar{u}| g(\bar{u} / \varrho)}{(\bar{u} / \varrho) g(\bar{u} / \varrho)} d x \\
& \leq \frac{1}{\varrho} \int_{B(\varrho)} \frac{|D \bar{u}| g(\bar{u} / \varrho)}{G(\bar{u} / \varrho)} d x \leq \frac{1}{\varrho} \int_{B(2 \varrho)} \frac{|D \bar{u}| g(\bar{u} / \varrho)}{G(\bar{u} / \varrho)} \eta^{g_{0}} d x .
\end{aligned}
$$

Using Young's inequality to estimate the numerator in this integral and then (2.8) gives $(2.7)$. give

In general, $p$ in Theorem 2.2 is very small, but the proof is easily modified to

$$
\left(R^{-n} \int_{B(2 R / 3)} G\left(\frac{u}{R}\right)^{p} d x\right)^{1 / p} \leq C_{p}\left[\inf _{B(R / 2)} G\left(\frac{u}{R}\right)+G(\chi)\right]
$$

for any $p \in(0, n /(n-1))$, in particular for $p=1$.

Standard arguments give Hölder continuity of solutions (see, e.g., [15, Theorem 8.22]). 
Corollary 2.3. If $Q u=0$ in $B(R)$, if $|u| \leq M$ in $B(R)$ and if the structure conditions (2.4a) and

$$
|B| \leq b_{0}|p| g(|p|)+b_{2}
$$

hold in $B(R)$, then there is a positive constant $\alpha$ determined only by $b_{0}, M, \delta, g_{0}$, and $n$ such that $u \in C^{\alpha}(B(R))$.

The preceding results are readily extended to the case that $Q u \geq 0$ (resp. $Q u \leq 0, Q u=0)$ in $B(R) \cap \Omega$ for suitable domains $\Omega$ (Lipschitz domains are included). In this case, $u$ must satisfy an appropriate boundary condition.

The results of DiBenedetto and Trudinger [11] for functions in De Giorgi classes can be modified along similar lines to show that functions in De Giorgitype classes based on our non-power functions satisfy corresponding inequalities. Hence quasi-minima and minima of the functional $\mathcal{I}$, from the introduction, are bounded and Hölder continuous if there are a function $b \in L^{q}$ for $q>n$ and a constant $\mu \geq 1$ such that

$$
G(|p|)-b(x)[G(|z|)+1] \leq f(x, z, p) \leq \mu G(|p|)+b(x)[G(|z|)+1] .
$$

3. Higher regularity. By modifying ideas set down by DiBenedetto [4] and Tolksdorf [35], it was shown in [24] that a large class of degenerate equations have smooth solutions. Specifically, let $F$ be a positive continuous function on $(0, \infty)$ such that

$$
F(t) \geq c_{1} F(4 t), \quad c_{2} F(t) t \leq F(s) s
$$

for all $s \geq t>0$ and let $A$ be a $C^{1}$ vector valued function on $\mathbb{R}^{n}$ such that $a^{i j}=\partial A^{i} / \partial p_{j}$ satisfies

$$
\left(a^{i j}(p)\right) \geq F(|p|) I, \quad\left|a^{i j}(p)\right| \leq \Lambda F(|p|), \quad|A(p)| \leq \Lambda|p| F(|p|) .
$$

(Here $c_{1}, c_{2}$, and $\Lambda$ are positive constants.) If $u$ is a weak solution of

$$
\operatorname{div} A(D u)=0 \quad \text { in } B(R),
$$

then $D u$ is Hölder continuous in $B(R)$ with exponent $\beta$ determined only by $c_{1}$, $c_{2}, \Lambda$, and $n$.

Certainly, if $g \in C^{1}$ satisfies (0.2), the $F$ defined by $F(t)=g(t) / t$ satisfies (3.1) with $c_{1}=4^{1-g_{0}}$ and $c_{2}=1$. Conversely, if $F$ satisfies (3.1) with $c_{1} \leq 4$ and $c_{2} \leq 1$ we can find a function $g$ satisfying $(0.2)$ with $\delta=0$ and $g_{0}=1-\log _{4} c_{1}$ (i.e. $c_{1}=4^{1-g_{0}}$ ) such that $c_{2} c_{1} g(t) \leq 4 t F(t)$ and $c_{1} t F(t) \leq 4 g(t)$. To construct $g$, first define $f(s)=\sup _{t \leq s} t F(t)$. Then $f$ is increasing,

$$
f(t) \geq \frac{1}{4} c_{1} f(4 t) \text { and } t F(t) \leq f(t) \leq t F(t) / c_{2} .
$$

Next define $h(t)=\log _{4} f\left(4^{t}\right)$, so $h$ is increasing and

$$
h(t-1)=\log _{4} f\left(\frac{1}{4} \cdot 4^{t}\right) \geq-1+\log _{4} c_{1}+h(t) .
$$

Now set $\phi(t)=\max \left\{0, \frac{1}{2}(1-|t|)\right\}$ and $k(t)=\int_{-\infty}^{\infty} h(t+s) \phi(s) d s$. (The crucial properties of $\phi$ are that $\int_{-\infty}^{\infty} \phi(s) d s=1, \phi \geq 0, \phi$ has support in $[-1,1], \phi(0)=\frac{1}{2}$ 
and $\phi^{\prime}$ changes sign only once, at 0.$)$ As $\phi$ is Lipschitz, $k$ is $C^{1}$ and a simple calculation gives

$$
\begin{aligned}
k^{\prime}(t) & =-\int_{-\infty}^{\infty} h(t+s) \phi^{\prime}(s) d s=\int_{-\infty}^{\infty}\{h(t)-h(t+s)\} \phi^{\prime}(s) d s \\
& =\int_{-1}^{0}\{h(t)-h(t+s)\} \phi^{\prime}(s) d s+\int_{0}^{1}\{h(t)-h(t+s)\} \phi^{\prime}(s) d s \\
& \leq \int_{-1}^{0}\{h(t)-h(t-1)\} \phi^{\prime}(s) d s+\int_{0}^{1}\{h(t)-h(t+1)\} \phi^{\prime}(s) d s \\
& \leq \int_{-1}^{0}\left\{1-\log _{4} c_{1}\right\} \phi^{\prime}(s) d s+\int_{0}^{1}\left\{1-\log _{4} c_{1}\right\} \phi^{\prime}(s) d s \\
& =\left(1-\log _{4} c_{1}\right) 2 \phi(0)=1-\log _{4} c_{1} .
\end{aligned}
$$

(For the inequalities, we use first that $h$ is increasing and then (3.4); we also take advantage of where $\phi^{\prime}$ is positive and where it is negative.) Defining $g(t)=$ $4^{k\left(\log _{4} t\right)}$, we see that $t g^{\prime}(t) / g(t)=k^{\prime}\left(\log _{4} t\right)$, and hence $(0.2)$ holds. In addition,

$$
\begin{array}{ll}
0 \leq h(t+s)-h(t) \leq 1-\log _{4} c_{1} & \text { if } 0 \leq s \leq 1, \\
0 \leq h(t)-h(t+s) \leq 1-\log _{4} c_{1} & \text { if }-1 \leq s \leq 0,
\end{array}
$$

and therefore $|k-h| \leq 1-\log _{4} c_{1}$, which implies that $c_{1} / 4 \leq g / f \leq 4 / c_{1}$.

In brief, $A$ satisfies (3.2) for some $F$ satisfying (3.1) if and only if $A$ satisfies

$$
\left(a^{i j}\right) \geq \frac{g(|p|)}{|p|} I, \quad\left|a^{i j}\right| \leq \Lambda g(|p|) /|p|,
$$

$$
|A| \leq \Lambda g(|p|)
$$

for some $g$ satisfying (0.2). Of course, the $\Lambda$ in (3.2) is not the same as in (3.5), but we do have $g_{0}=1-\log _{4} c_{1}$.

By combining a simple $L^{\infty}$ gradient bound with the basic estimates used to prove the $C^{1, \beta}$ regularity for weak solutions of (3.3), we obtain the following Campanato-type estimate in which $\{w\}_{R}$ denotes the mean value of $w \in$ $L^{1}(B(R))$.

THEOREM 3.1. Let $F$ be a positive continuous function on $(0, \infty)$ satisfying (3.1) and let $A$ be a $C^{1}$ function with range and domain $\mathbb{R}^{n}$ satisfying (3.2). Then there are positive constants $C$ and $\sigma$ depending only on $c_{1}, c_{2}, \Lambda$, and $n$ such that

$$
\int_{B(r)} G\left(\left|D u-\{D u\}_{r}\right|\right) d x \leq C\left(\frac{r}{R}\right)^{n+\sigma} \int_{B(R)} G\left(\left|D u-\{D u\}_{R}\right|\right) d x
$$

for all $r \in(0, R)$.

Pr o of. [26, Lemma 5.1]. 
From this estimate, a $C^{1, \beta}$ estimate follows under the appropriate analog (and generalization) of (1.3) via a perturbation argument based on the one used by Giaquinta and Giusti [14]. One advantage of this perturbation argument is that the conditions on $A_{x}$ and $A_{z}$ can be relaxed to Hölder conditions on $A$ with respect to $x$ and $z$.

TheOREM 3.2. Let $g$ satisfy (0.2) and suppose $A$ and $B$ satisfy (3.5a), and (3.7a) $|A(x, z, p)-A(y, w, p)| \leq \Lambda_{1}(1+g(|p|))\left[\left(1+|p|^{\alpha}\right)|x-y|^{\alpha}+|z-w|^{\beta}\right]$,

$$
|B(x, z, p)| \leq \Lambda_{1}(1+|p| g(|p|))
$$

for some constants $\Lambda_{1} \geq 0$ and $\alpha$ and $\beta$ in $(0,1]$. Then any Lipschitz weak solution of $Q u=0$ in $\Omega$ has a Hölder continuous gradient there. The Hölder exponent depends only on $\alpha, \beta, \Lambda, g_{0}$, and $n$, while the Hölder constant on a given subdomain $\Omega^{\prime} \Subset \Omega$ depends also on $\delta$, $\operatorname{dist}\left(\Omega^{\prime}, \partial \Omega\right), \Lambda_{1}$, and $\operatorname{osc}_{\Omega} u$, but not on the assumed bound on Du.

\section{Proof. [26, Section 5].}

Standard interpolation arguments give a bound on the gradient in Theorem 3.2; however, the finiteness of $\sup _{\Omega}|D u|$ is used in the proof. When (3.7a) is relaxed to the structure condition

$$
|A(x, z, p)-A(y, w, p)| \leq \Lambda_{1}(1+g(|p|))\left[|x-y|^{\alpha}+|z-w|^{\beta}\right],
$$

one can work directly with bounded weak solutions.

Corresponding boundary regularity results are proved rather easily. For the Dirichlet condition (1.4a), a well-known theorem of Krylov [19] gives regularity up to the boundary if $Q$ has the special form in Theorem 3.1, $\phi \in C^{1, \alpha}$ and $\partial \Omega$ is locally a hyperplane. For the conormal condition $(1.4 \mathrm{~b})$, the interior argument from Theorem 3.1 can be modified if also $\psi$ is constant (and $\partial \Omega$ is locally a hyperplane). Both these results are proved in [24]. Again the perturbation argument used in Theorem 3.2 applies (with some modification) to give boundary regularity under the full structure conditions (3.5a) and (3.7).

4. Extensions of the results. The previous sections give a fairly complete story of the operator $Q u$ when $A$ is roughly $f(|p|) p$ for a suitable function $f$. We now investigate some variations on this form.

First suppose $A^{i}=\left|D_{i} u\right|^{m-2} D_{i} u$ for some constant $m>1$ and $B \equiv 0$. Then the results of Ladyzhenskaya and Ural'tseva show that solutions of $Q u=0$ are locally bounded and Hölder continuous. It appears that these solutions are also locally Lipschitz, but $C^{1, \alpha}$ regularity is currently unknown. (I suspect it to be false in general.)

Another way in which our structure conditions can be modified is to introduce anisotropy. The model for this situation is $A^{i}=\left|D_{i} u\right|^{m_{i}-2} D_{i} u$ with different numbers $m_{1}, \ldots, m_{n}$ (but all $>1$ ). An important counterexample of Giaquinta [13] shows that solutions of anisotropic equations need not be smooth. Specifically, 
if $m_{i}=2$ for $i=1, \ldots, 5$ and $m_{6}=5$, then there are unbounded weak solutions of $\sum_{i=1}^{6} D_{i}\left(\left|D_{i} u\right|^{m_{i}-2} D_{i} u\right)=0$. Giaquinta also modifies this construction to provide unbounded solutions in any number of dimensions provided $m_{1}=\ldots=m_{n-1}$ and $m_{n}$ are sufficiently far apart. On the other hand, Korolev [17] proved a global bound (based on the boundary maximum) for a general class of anisotropic equations: Let $g_{i}$ be $n$ functions satisfying (0.2) and define functions $\phi_{i}, P, H$, and $\bar{H}$ by

If

$$
\begin{aligned}
\phi_{i}(z) & =\sup _{s>0} \frac{g_{i}(z s)}{g_{i}(s)}, & P^{-1}(z)=\left(\prod_{i=1}^{n} \phi_{i}^{-1}(z)\right)^{1 / 2}, \\
H(z) & =1 / P(1 / z), & \bar{H}^{-1}(z)=z / H^{-1}(z) .
\end{aligned}
$$

$$
p \cdot A \geq \sum_{i=1}^{n} g_{i}\left(p_{i}\right)-H(u), \quad|B| \leq \bar{H}^{-1}\left(\sum_{i=1}^{n} g_{i}\left(p_{i}\right)\right)+H(u) / u+C
$$

and if some technical assumptions are met (for example, $D_{i} u \in L^{G_{i}}$, and the functions $g_{i}$ do not increase so rapidly that $u$ is bounded by a Sobolev type embedding), then any solution $u$ of

$$
\operatorname{div} A(x, u, D u)+B(x, u, D u)=0 \quad \text { in } \Omega, \quad u=\phi \quad \text { on } \partial \Omega,
$$

with $\phi \in L^{\infty}$, is bounded, and the $L^{\infty}$ norm of $u$ can be estimated in terms of quantities involving the $g_{i}$ 's, $n, \Omega, C$, and the $L^{\infty}$ norm of $\phi$. (In fact, the conditions

$$
p \cdot A \geq \sum g_{i}\left(p_{i}\right)-P(u), \quad u B \leq \sum g_{i}\left(p_{i}\right)+P(u)+C
$$

suffice for Korolev's proof.) Korolev also proves a local $L^{\infty}$ bound if the additional conditions

$$
\begin{gathered}
\sum \bar{g}_{i}\left(A^{i}\right) \leq \sum g_{i}\left(p_{i}\right)+g^{*}(z), \\
\lim _{t \rightarrow \infty} \frac{g_{i}(k t)}{g^{*}(t)}=0 \quad \text { for all } k>0, i=1, \ldots, n,
\end{gathered}
$$

are satisfied, where $\bar{g}_{i}$ and $g^{*}$ are defined by $\bar{g}_{i}^{-1}(z)=z / g_{i}^{-1}(z), g^{*-1}(z)=$ $\int_{0}^{|z|} t^{-1-1 / n}\left(\prod g_{i}^{-1}(t)\right)^{1 / n} d t$. Note that in case the $g_{i}$ 's are power functions, $(4.2 \mathrm{~b})$ restricts the distribution of the exponents. This same restriction occurs in the global bound of Boccardo, Marcellini, and Sbordone [1] for solutions of (4.1) when $B=0$ and $A^{i}=\left|D_{i} u\right|^{m_{i}-2} D_{i} u-f^{i}$ with $f^{i} \in L^{r_{i}}$. Setting $q=n / \sum m_{i}, q_{i}=$ $m_{i}\left(m_{1}-1\right)$, they prove an $L^{\infty}$ estimate for $u$ provided $r_{i}>q_{i}$ for all $i$ and

$$
\frac{n q}{n-q} / \max _{i} m_{i}>\max _{i} \frac{r_{i}}{r_{i}-q_{i}} .
$$

Our results can also be extended to solutions of obstacle type variational inequalities rather than elliptic differential equations. Here $\psi$, the obstacle, is 
some function with $\psi \leq \phi$ on $\partial \Omega$, we define

$$
\mathbb{K}=\left\{v \in W^{1, G}: v=\phi \text { on } \partial \Omega, v \geq \psi \text { in } \Omega\right\},
$$

and we seek a function $u \in \mathbb{K}$ such that

$$
\int A(x, u, D u) \cdot D(u, v)-B(x, u, D u)(u-v) d x \leq 0
$$

for all $v \in \mathbb{K}$. Standard convex analysis provides criteria guaranteeing existence and uniqueness of solutions, so we only discuss regularity. If $\psi$ is locally continuous, so is $u$ and the moduli of continuity $\omega_{\psi}$ and $\omega_{u}$ are related by $\omega_{u}(r) \leq C\left(\omega_{\psi}(r)+r^{\alpha}\right)$ for the $\alpha$ from Corollary 2.3. The power case was proved by Michael and Ziemer [30] and the general case by Lieberman [27], who proved a similar boundary estimate. Michael and Ziemer actually assume less of the obstacle than continuity, and they prove a boundary estimate in [31]. If $\psi \in C^{1, \sigma}$ for $\sigma$ sufficiently small (depending on $\alpha, \beta, \Lambda, g_{0}$, and $n$ as in Theorem 3.2), then $u \in C^{1, \sigma}$. The power case was proved by $\mathrm{Mu}[32]$, and Choe [2] (under slightly stronger hypotheses) and the general case by Lieberman [27]. All three works also discuss boundary regularity. Choe [3], Kilpeläinen and Ziemer [16], Lieberman [28] and $\mathrm{Mu}$ and Ziemer [33] also consider a 2-obstacle problem, in which the inequalities $\psi \leq \phi$ and $v \geq \psi$ are replaced by $\psi_{1} \leq \phi \leq \psi_{2}$ and $\psi_{2} \geq v \geq \psi_{1}$, respectively, in the definition of $\mathbb{K}$. Choe, Lieberman, and $\mathrm{Mu}$ all worked independently and almost simultaneously, using three quite different methods.

The next generalization is to parabolic equations instead of elliptic ones. Here the situation is noticeably different and most results are known only in the power case $g(t)=t^{m-1}$. If $m>2 n /(n+1)$, then weak solutions are bounded; otherwise they are generally unbounded [10]. However, global $L^{\infty}$ estimates (in terms of boundary and initial data) are valid [22, Theorem 4.1].

DiBenedetto [5] showed that bounded solutions are Hölder continuous for $m \geq 2$ (in fact the case $m=2$ is due to Ladyzhenskaya and Ural'tseva [20]). With Chen [6], he showed the same thing for $1<m<2$ provided $A$ is independent of $t$. The parabolic Hölder estimate is trickier than the elliptic one because of a qualitative difference in the behavior of solutions. In particular, if $m>2$, then there are self-similar solutions of $\operatorname{div}\left(|D u|^{m-2} D u\right)=u_{t}$ which have compact support at any fixed time [5], and if $m<2$, all solutions of $\operatorname{div}\left(|D u|^{m-2} D u\right)=u_{t}$ must be identically zero after some finite time [6]. Such behavior precludes the possibility of a weak Harnack inequality.

Gradient estimates (for sufficiently smooth solutions) hold even in the nonpower case, based on bounds on the solution and, sometimes, continuity estimates as well. The general interior estimate was proved in [22] and a boundary gradient estimate for Dirichlet boundary data appears in [23, Theorem 2.2]. In both cases, $\alpha=\beta=1$ in condition (3.7a).

Hölder gradient estimates in the power case were first proved by DiBenedetto and Friedman [8,9] and by Wiegner [38]. Both works were concerned with systems, so their results, when specialized to a single equation, are not quite as general as 
others quoted here. For the general, non-power case, Hölder gradient estimates were proved by Lieberman [25] under the special structure of Theorem 3.1 and for the full structure only in the power case. Combining the parabolic perturbation arguments of [25] with the general structure perturbation argument of [26] should produce the parabolic analog of Theorem 3.2. An interesting aspect of these results is that $A$ is allowed to depend on $t$, but this dependence need not be continuous. Thus, initial regularity is proved very easily: Suppose $u$ solves

$$
\begin{aligned}
u_{t} & =\operatorname{div} A(x, t, u, D u)+B(x, t, u, D u) \quad \text { in } \Omega \times(0, T), \\
u & =u_{0} \quad \text { in } \Omega \times\{0\}
\end{aligned}
$$

with $u_{0} \in C^{1, \alpha}$. We define $u, A$ and $B$ for negative $t$ 's by

$$
A(x, t, z, p)=p-D u_{0}(x), \quad B \equiv 0, \quad u(x, t)=u_{0}(x, 0) .
$$

Then $u$ is a weak solution of

$$
u_{t}=\operatorname{div} A(x, t, u, D u)+B(x, t, u, D u) \quad \text { in } \Omega \times(-T, T)
$$

and interior estimates in $\Omega \times(-T, T)$ imply the corresponding initial estimates in $\Omega \times(0, T)$.

Parabolic boundary estimates are also more complicated. The Hölder estimates of [5] and [6] extend easily to the boundary. Hölder gradient bounds for conormal boundary conditions follow from the interior estimates as before [25], and Hölder gradient bounds for Dirichlet data (which were not known when these lectures were given) have recently been proved in [29]. DiBenedetto and Chen [7] show that solutions of such equations (or systems) with $C^{1, \alpha}$ Dirichlet boundary data are Hölder continuous in the parabolic metric with exponent less than one. For a single equation, this result is weaker than the boundary gradient estimate of $[23]$.

\section{References}

[1] L. Boccardo, P. Marcellini and C. Sbordone, $L^{\infty}$-regularity for variational problems with sharp non standard growth conditions, Boll. Un. Mat. Ital. (7) 4-A (1990), 219-225.

[2] H. J. Choe, A regularity theory for a more general class of quasilinear elliptic differential equations and obstacle problems, Arch. Rational Mech. Anal. 114 (1991), 393-394.

[3] —, Regularity for certain degenerate elliptic double obstacle problems, J. Math. Anal. Appl., to appear.

[4] E. DiBenedetto, $C^{1+\alpha}$ local regularity of weak solutions of degenerate elliptic equations, Nonlinear Anal. 7 (1983), 827-850.

[5] - - On the local behavior of solutions of degenerate parabolic equations with measurable coefficients, Ann. Scuola Norm. Sup. Pisa (4) 13 (1986), 487-535.

[6] E. DiBenedetto and Y.-Z. Chen, On the local behaviour of solutions of singular parabolic equations, Arch. Rational Mech. Anal. 103 (1988), 319-345.

[7] - - - Boundary estimates for solutions of nonlinear degenerate parabolic systems, J. Reine Angew. Math. 395 (1989), 102-131. 
[8] E. DiBenedetto and A. Friedman, Regularity of solutions of nonlinear degenerate parabolic systems, ibid. 349 (1984), 83-128.

[9] - , - Hölder estimates for nonlinear degenerate parabolic systems, ibid. 357 (1985), 1-22.

[10] E. DiBenedetto and M. A. Herrero, Non-negative solutions of the evolution p-Laplacian equation. Initial traces and Cauchy problem when $1<p<2$, Arch. Rational Mech. Anal. 111 (1990), 225-290.

[11] E. DiBenedetto and N. S. Trudinger, Harnack inequalities for quasi-minima of variational integrals, Ann. Inst. H. Poincaré Anal. Non Linéaire 1 (1984), 295-308.

[12] T. K. Donaldson and N. S. Trudinger, Orlicz-Sobolev spaces and imbedding theorems, J. Funct. Anal. 8 (1971), 52-75.

[13] M. Giaquinta, Growth conditions and regularity, a counterexample, Manuscripta Math. 59 (1987), 245-248.

[14] M. Giaquinta and E. Giusti, Global $C^{1, \alpha}$ regularity for second order quasilinear elliptic equations in divergence form, J. Reine Angew. Math. 351 (1984), 55-65.

[15] D. Gilbarg and N. S. Trudinger, Elliptic Partial Differential Equations of Second Order, 2nd ed., Springer, Berlin 1983.

[16] T. Kilpeläinen and W. P. Ziemer, Pointwise regularity of solutions to nonlinear double obstacle problems, Ark. Mat. 29 (1991), 83-106.

[17] A. G. Korolev, On boundedness of generalized solutions of elliptic differential equations with nonpower nonlinearities, Mat. Sb. 180 (1989), 78-100 (in Russian); English transl.: Math. USSR-Sb. 66 (1990), 83-106.

[18] M. A. Kranosel'skii and Ya. B. Rutickii, Convex Functions and Orlicz Spaces, Noordhoff, Groningen 1961.

[19] N. V. Krylov, Boundedly nonhomogeneous elliptic and parabolic equations in a domain, Izv. Akad. Nauk SSSR Ser. Mat. 47 (1983), 75-108 (in Russian); English transl.: Math. USSR-Izv. 21 (1984), 67-98.

[20] O. A. Ladyženskaja, V. A. Solonnikov and N. N. Ural'ceva, Linear and Quasilinear Equations of Parabolic Type, Amer. Math. Soc., Providence, R.I., 1967.

[21] O. A. Ladyzhenskaya and N. N. Ural'tseva, Linear and Quasilinear Elliptic Equations, Nauka, Moscow 1964 (in Russian); English transl.: Academic Press, New York 1968. 2nd Russian ed., 1973.

[22] G. M. Lieberman, Interior gradient bounds for non-uniformly parabolic equations, Indiana Univ. Math. J. 32 (1983), 579-601.

[23] —, The first initial-boundary value problem for quasilinear second order parabolic equations, Ann. Scuola Norm Sup. Pisa (4) 13 (1986), 347-387.

[24] - Boundary regularity for solutions of degenerate elliptic equations, Nonlinear Anal. 12 (1988), 1203-1219.

[25] - Boundary regularity for solutions of degenerate parabolic equations, ibid. 14 (1990), $501-524$.

[26] —, The natural generalization of the natural conditions of Ladyzhenskaya and Ural'tseva for elliptic equations, Comm. Partial Differential Equations 16 (1991), 311-361.

[27] - Local and boundary regularity for some variational inequalities involving p-Laplaciantype operators, to appear.

[28] —, Regularity of solutions to some degenerate double obstacle problems, Indiana Univ. Math. J. 40 (1991), 1009-1028.

[29] —, Boundary and initial regularity for solutions of degenerate parabolic equations, Nonlinear Anal., to appear.

[30] J. H. Michael and W. P. Ziemer, Interior regularity for solutions to obstacle problems, Nonlinear Anal. 10 (1986), 1427-1448.

[31] —, Existence of solutions to obstacle problems, ibid. 17 (1991), 45-71. 
[32] J. Mu, Higher regularity of the solution to the p-Laplacian obstacle problem, J. Differential Equations 95 (1992), 370-384.

[33] J. Mu and W. P. Ziemer, Smooth regularity of solutions of double obstacle problems involving degenerate elliptic equations, Comm. Partial Differential Equations 16 (1991), 821-843.

[34] L. M. Simon, Interior gradient bounds for non-uniformly elliptic equations, Indiana Univ. Math. J. 25 (1976), 821-855.

[35] P. Tolksdorf, Regularity for a more general class of quasilinear elliptic equations, J. Differential Equations 51 (1984), 126-150.

[36] K. Uhlenbeck, Regularity for a class of non-linear elliptic systems, Acta Math. 138 (1977), 219-240.

[37] N. N. Ural'tseva, Degenerate quasilinear elliptic systems, Zap. Nauchn. Sem. Leningrad. Otdel. Mat. Inst. Steklov. (LOMI) 7 (1968), 184-222 (in Russian); English transl.: Sem. Math. V. A. Steklov Math. Inst. Leningrad 7 (1968), 83-99.

[38] M. Wiegner, On $C_{\alpha}$-regularity of the gradient of solutions of degenerate parabolic systems, Ann. Mat. Pura Appl. 145 (1986), 385-405. 